\title{
Zur Kenntnis des Dianthryls.
}

\section{Dritte Mitteilung über Zweikernchinone ${ }^{1}$}

von

\section{Alfred Eckert und Alice Hofmann.}

Aus dem chemischen Laboratorium der k. k. Deutschen Universität in Prag.

(Vorgelegt in der Sitzung am 29. April 1915.)

Der dem Dianthranol:
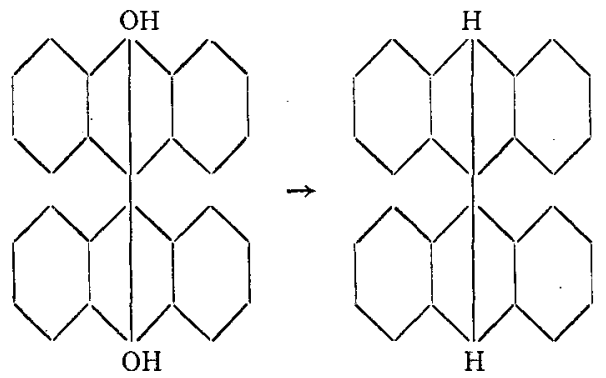

entsprechende Kohlenwasserstoff, das Dianthryl, wurde von Liebermann und Gimbl2 durch Reduktion von Anthrachinon mit Zinn und Salzsäure in Eisessiglösung dargestellt. In vorliegender Arbeit wurde versucht, einerseits das Dianthryl zu Dianthranol zu oxydieren, andrerseits durch Reduktion vom Dianthranol zum Dianthryl zu gelangen. K. H. Meyer ${ }^{3}$

1 Erste Mitteilung: Hans Meyer, Monatshefte für Chemie, 30, 165 (1909). - Zweite Mitteilung: Hans Meyer, R. Bondy und A. Eckert, Monatshefte für Chemie, 33, 1447 (1912).

2 Berl. Ber., 20, 1854 (1887).

3 Annalen, 379, 75 (1911). 
hat durch oxydierende Acetylierung Anthracen zu Anthranolacetat oxydiert:

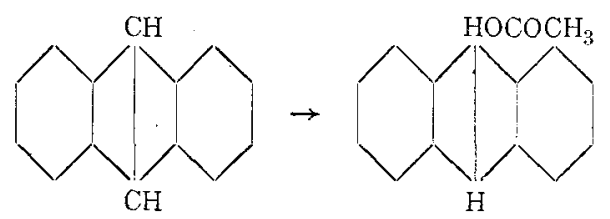

Nach derselben Methode gelang auch die Überführung des Dianthryls in Dianthranolacetat:

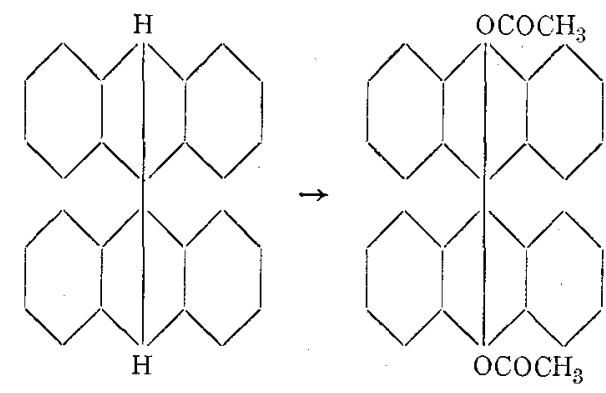

Der Versuch, Dianthryl mit Chromsäure in Eisessiglösung:
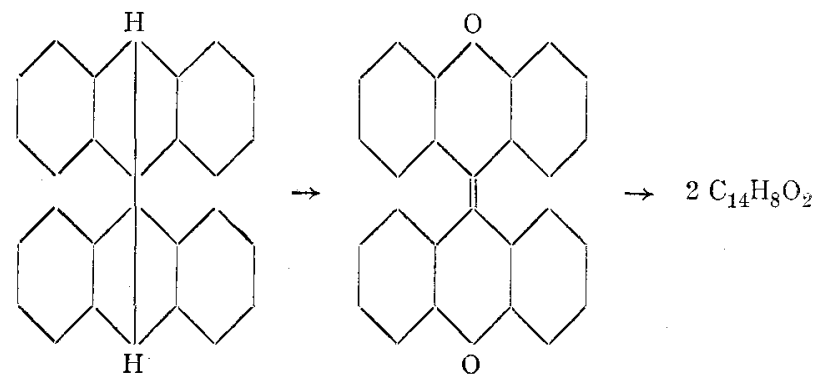

zu Bianthron zu oxydieren, gelang nicht. Als einziges Reaktionsprodukt konnte Anthrachinon isoliert werden.

Behandelt man Dianthranol mit Zinn und Salzsäure in Eisessiglösung, so erhält man leicht und in guter Ausbeute Dianthryl: 

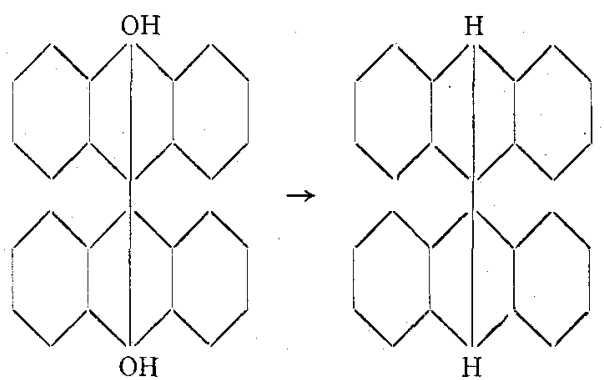

Verwendet man an Stelle des Dianthranols das isomere Dihydrobianthron, so erhält man als primäres Reaktionsprodukt eine sehr labile Substanz, die durch Wasserabspaltung leicht in Dianthryl überführt werden konnte. Die Analyse dieser Substanz lieferte Werte, die auf ein 10,10'-Dioxytetrahydrodianthryl stimmten:

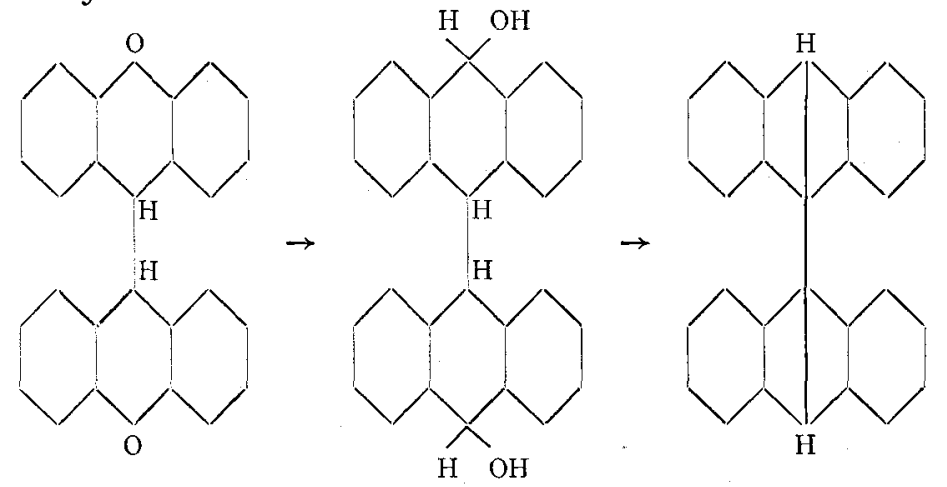

Eine Substanz derselben Zusammensetzung, das sogenannte Anthrapinakon (nach der oben angewendeten Bezeichnung wäre diese Substanz als 9, 9'-Dioxytetrahydrodianthryl $\mathrm{zu}$ benennen):

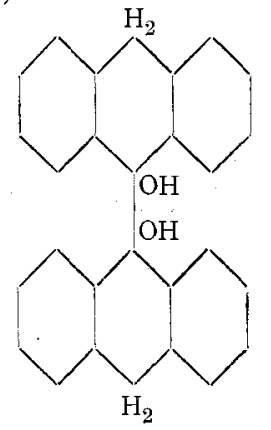


hat Schulze ${ }^{1}$ durch Reduktion von Anthrachinon mit Ammoniak und Zinkstaub dargestellt. Auch das Anthrapinakon liefert durch Wasserabspaltung Dianthryl. Während man aber bei Verwendung von Anthrapinakon ziemlich kräftige, wasserentziehende Mittel (Acetylchlorid) verwenden muß, um Dianthryl zu erhalten, spaltet unsere Substanz äußerst leicht Wasser ab. Wie wir gefunden haben, kann man Anthrapinakon bei Vermeidung allzu langen Kochens unverändert aus Essigsäureanhydrid umkrystallisieren. Unsere Substanz spaltet schon bei längerem Kochen mit Alkohol oder Eisessig oder beim Umkrystallisieren aus Essigsäureanhydrid Wasser ab und geht in Dianthryl über.

Die angenommene Konstitution des Anthrapinakons und des 10,10'-Dioxytetrahydrodianthryls, die schon aus ihrer Darstellung sehr wahrscheinlich wird, konnte durch die Oxydation sichergestellt werden. Hierbei lieferte das Schulze'sche Anthrapinakon Anthrachinon, das 10,10'-Dioxytetrahydrodianthryl Dihydrobianthron.

\section{Experimenteller Teil. \\ Zur Darstellung des Dianthryls.}

Die Darstellung von Dianthryl nach den Angaben von Liebermann und Gimbl wollte uns ursprünglich nicht recht gelingen. Recht gute Ausbeuten erhält man, wenn man der Reduktionslösung eine Spur Platinchlorid zusetzt. Wir haben folgendermaßen gearbeitet: $40 \mathrm{~g}$ Anthrachinon, $200 \mathrm{~cm}^{3}$ Eisessig, $80 \mathrm{~g}$ Zinn und 2 bis 3 Tropfen einer verdünnten Platinchloridlösung werden zum Sieden erhitzt und im Verlauf einer halben Stunde $100 \mathrm{~cm}^{3}$ konzentrierter Salzsäure in Portionen zu $10 \mathrm{~cm}^{3}$ eingetragen. Man erhitzt noch eine Stunde zum Sieden, filtriert $a b$ und krystallisiert aus Toluol um.

\section{Oxydation von Dianthryl.}

$1.7 \mathrm{~g}$ fein gepulvertes Dianthryl werden in $200 \mathrm{~cm}^{3}$ Eisessig suspendiert. Man trägt nun in diese Suspension bei

1 Berl. Ber., 18, 3034 (1885). 
50 bis $70^{\circ}$ unter häufigem Umschütteln im Verlauf einer Stunde $2.6 \mathrm{~g}$ fein gepulvertes Bleidioxyd ein. Hierauf wird mit Wasser gefällt. Der Niederschlag wird mit Aceton ausgekocht, wobei unverändertes Dianthryl und Bleidioxyd zurückbleiben. Nach einigem Stehen krystallisiert aus der Acetonlauge die Substanz in feinen Nädelchen, die nach dem Umkrystallisieren aus Aceton und Eisessig bei $284^{\circ}$ schmelzen. Ein Mischungsschmelzpunkt mit Dianthranolacetat ergab keine Depression. Die Ausbeuten an Dianthranolacetat waren sehr gut und das Produkt sehr rein. Zur näheren Charakterisierung des erhaltenen Dianthranolacetats wurde versucht, dasselbe durch Verseifen in Dianthrol zu überführen. Erwärmt man Dianthranolacetat kurze Zeit mit konzentrierter Schwefelsäure am Wasserbad, so schlägt die ursprünglich braungrüne Farbe der Lösung in Rotviolett um, gleichzeitig entweicht Schwefeldioxyd. Es hatte also neben der Verseifung gleichzeitig Oxydation des Dianthranols zu Dianthron, das sich in konzentrierter Schwefelsäure rotviolett löst, stattgefunden. Da diese Beobachtung immerhin etwas merkwürdig ist, wurde reines Dianthranol in konzentrierter Schwefelsäure gelöst und am Wasserbad erwärmt. Schon nach kurzer Zeit tritt der Geruch nach Schwefeldioxyd auf und die Lösung wird violett. Es gelingt jedoch nicht, auf diese Weise reines Dianthron darzustellen, da die Oxydation nicht bei dieser Stufe stehen bleibt, sondern weitergeht.

Da die saure Verseifung des Dianthranolacetats nicht gelang, wurde alkalisch verseift. $2 \mathrm{~g}$ Dianthranolacetat wurden mit $50 \mathrm{~cm}^{3}$ Alkohol und $10 \mathrm{~cm}^{3} 2$ normaler Kalilauge 3 Viertelstunden gekocht. Die erhaltene dunkelrote Lösung wurde mit Wasser verdünnt und sofort in Salzsäure einfiltriert. Man erhält auf diese Weise Dianthranol mit allen von Hans Meyer angegebenen Eigenschaften. Zur näheren Charakterisierung wurde es auch noch durch Belichtung in Mesonaphthodianthron überführt, das durch seine Schwerlöslichkeit in allen organischen Lösungsmitteln und durch die Reaktion mit konzentrierter Schwefelsäure leicht identifiziert werden konnte. 


\section{Reduktion von Dianthranol.}

$2 g$ Dianthranol, $50 \mathrm{~cm}^{3}$ Eisessig, $5 g$ Zinnspäne und eine Spur Platinchlorid wurden zum Sieden erhitzt und in die siedende Lösung nach und nach $25 \mathrm{~cm}^{3}$ konzentrierte Salzsäure im Verlauf einer Stunde eingetragen. Man erhält kurze Zeit im Sieden und filtriert heiß ab. Nach dem Umkrystallisieren aus Essigsäure oder Toluol schmilzt die Substanz bei $300^{\circ}$ und zeigt alle Reaktionen des Dianthryls von Liebermann. Ein Gemisch der so dargestellten Substanz mit reinem Dianthryl schmilzt ebenfalls bei $300^{\circ}$.

\section{Reduktion von Dihydrodianthron.}

$5 \mathrm{~g}$ Dihydrodianthron wurden in $50 \mathrm{~cm}^{3}$ Eisessig gelöst und mit etwas Platinchlorid versetzt. In die siedende Lösung trugen wir $10 \mathrm{~g}$ Zinnspäne und im Verlauf von 2 Stunden $25 \mathrm{~cm}^{3}$ rauchende Salzsäure ein. Die ursprünglich helle Lösung färbte sich nach und nach dunkel, wurde aber nach einiger Zeit wieder fast vollkommen farblos. Nach dem Erkalten wurde mit Wasser verdünnt, abfiltriert. Der Rückstand wurde mehrere Male mit Petroläther ausgekocht. Beim Erkalten krystallisierten aus dem Petroläther schöne gelbliche Nadeln, die bei 150 bis $153^{\circ}$ schmelzen. Die Analyse ergibt:

6.328 $m g$ geben $19 \cdot 92 m g \mathrm{CO}_{2}, 3 \cdot 32 m g \mathrm{H}_{2} \mathrm{O}$.

$$
\begin{aligned}
\text { Gefunden: } & \mathrm{C} \ldots \ldots 85 \cdot 81 \% \\
& \mathrm{H} \ldots \ldots 5 \cdot 86 \% \\
\text { Für } \mathrm{C}_{28} \mathrm{H}_{22} \mathrm{O}_{2} \text { berechnet: } & \mathrm{C} \ldots \ldots 86 \cdot 15 \% \\
& \mathrm{H} \ldots \ldots 5 \cdot 64 \%
\end{aligned}
$$

Das Dioxytetrahydrodianthryl ist leicht löslich in den gebräuchlichen Lösungsmitteln und krystallisiert am besten aus Petroläther. Beim Sublimieren oder Umkrystallisieren aus Essigsäureanhydrid tritt Wasserabspaltung ein unter Bildung von Dianthryl. Auch durch längeres Kochen mit Alkohol oder Eisessig kann man die Substanz in Dianthryl überführen. 


\section{Oxydation \\ von Anthrapinakon und Dioxytetrahydrodianthryl.}

Anthrapinakon wurde in Eisessig gelöst und zur siedenden Lösung eine wässerige Lösung von Ammoniumpersulfat zugesetzt. Nach kurzem Kochen verdünnt man mit Wasser, filtriert $a b$ und krystallisiert aus Eisessig um. Die Substanz erweist sich als reines Anthrachinon, was durch seinen Schmelzpunkt und das Verhalten gegen Zink und Lauge leicht ermittelt werden kann. Behandelt man Dioxytetrahydrodianthryl in gleicher Weise (wobei man aber die Substanz nicht in heißem, sondern nur in kaltem Eisessig lösen darf), so erhält man Dihydrodianthron, das durch den Schmelzpunkt, seine leichte Enolisierbarkeit leicht als solches identifiziert werden kann. 